\title{
EDITORIAL
}

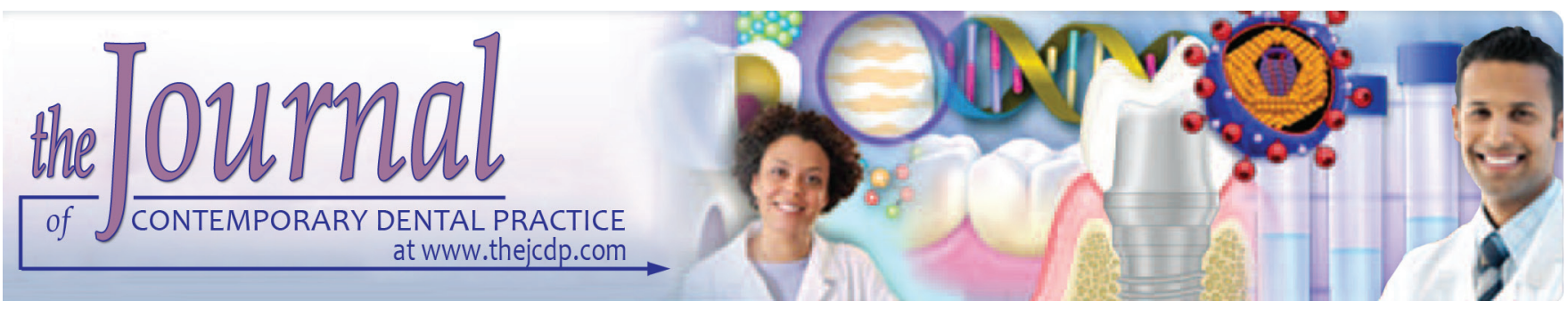

\section{Aligners: the Invisible Corrector-A Boon or Bane}

\author{
Lodd Mahendra
}

How to cite this article: Mahendra L. Aligners: the Invisible Corrector-A Boon or Bane. J Contemp Dent Pract 2018;19(3):247.

\section{Source of support: Nil}

\section{Conflict of interest: None}

The trend of clinical orthodontics has shown a palpable shift from conventional braces to innovative technologies like invisible aligners. Aligners are sequences of clear trays worn by patients to straighten their teeth. They were envisaged for the main purpose of esthetics, mainly directed toward self-conscious teenagers who otherwise would shy away from essential correction of malocclusion.

With increased awareness for orthodontic treatment among young adults, there arose an increased need for esthetic orthodontic correction. Peer pressure and social stigma resulting from metal braces have for long been a drawback of standard therapy. Though ceramic braces had been esthetically superior to metal appliances, the rise of clear aligners has made orthodontic correction more unnoticeable and attractive. Furthermore, the advent of scanning technology has made clear aligners commercialized and less dependent on human intervention (orthodontists). However, the applications of clear aligners are limited as they have been proved to be effective only in the correction of mild cases of malocclusion.

There have been several long-term studies of the efficacy, biocompatibility, and adverse effects of conventional orthodontics. Only recently various studies have been conducted to analyze the effect of clear aligners on patients. A systematic review of literature conducted to determine

Department of Orthodontics, Sri Venkateswara Dental College and Hospital, Chennai, Tamil Nadu, India

Corresponding Author: Lodd Mahendra, Department of Orthodontics, Sri Venkateswara Dental College and Hospital Chennai, Tamil Nadu, India, e-mail: loddmahendra@gmail.com the effect of aligners showed only limited level of evidence in favor of aligners. ${ }^{1}$ Furthermore, the increasing trend toward marketing these products and making them available over the counter without the guidance of a qualified dental professional raises ethical and moral concerns.

Though the promotion of these products reflects the evolution in the field of orthodontics, the limitations pertaining to this treatment and adverse reactions related to the use of these materials should be taken into consideration. A retrospective analysis conducted by the Manufacturer and User Facility Device Experience database of the United States Food and Drug Administration ${ }^{2}$ recorded reports of individual adverse clinical events like difficulty in breathing, sore throat, swollen tongue, itchiness, swollen gums, etc. ${ }^{3}$ The study has its own limitations, but the clinical observations cannot be ignored.

A well-qualified professional should be the right person to identify the indicated patient and should possess adequate knowledge and experience to explain to the patient regarding the various therapeutic options, including the above products. They should also be aware of such potential adverse effects and address such events as and when reported.

Though technology and commerce have evolved greatly in the field of medicine, limitations do exist. This field of dentistry requires a conscientious practitioner to decide the best for the patient.

\section{REFERENCES}

1. Lagravère MO, Flores-Mir $\mathrm{C}$. The treatment effects of Invisalign orthodontic aligners. A systematic review. 2005 Dec;136(12):1724-1729.

2. U.S. Food and Drug administration. Manufacturer and user facility device experience (MAUDE). Available at https:// www.accessdata.fda.gov/scripts/cdrh/cfdocs/cfmaude/ search.cfm. Accessed December 14, 2016.

3. Allareddy V, Nalliah R, Lee MK, Rampa S, Allareddy V. Adverse clinical events reported during Invisalign treatment: Analysis of the MAUDE database. Am J Orthod Dentfacial Orthop 2017 Nov;152(5):706-710. 